\title{
Short-Distance Atomic Beam Deceleration with a Stimulated Light Force
}

\author{
J. Söding, ${ }^{1}$ R. Grimm, ${ }^{1}$ Yu. B. Ovchinnikov, ${ }^{1}$ Ph. Bouyer, ${ }^{2, *}$ and Ch. Salomon ${ }^{2}$ \\ ${ }^{1}$ Max-Planck-Institut für Kernphysik and Physikalisches Institut der Universität Heidelberg, 69029 Heidelberg, Germany \\ ${ }^{2}$ Laboratoire Kastler Brossel, Ecole Normale Supérieure, 24 Rue Lhomond, 75231 Paris Cedex 05, France
}

(Received 23 September 1996)

\begin{abstract}
We have decelerated a cesium atomic beam from thermal velocities down to several tens of $\mathrm{m} / \mathrm{s}$ within only a $10 \mathrm{~cm}$ slowing distance. A bichromatic standing light wave was used to generate a stimulated force exceeding the spontaneous force limit by a factor of $\sim 10$ and extending over a large, saturation-broadened velocity range. Because of the short slowing distance this method allows production of very intense, continuous beams of slow atoms. [S0031-9007(97)02492-7]
\end{abstract}

PACS numbers: $32.80 . \mathrm{Pj}, 42.50 . \mathrm{Vk}$

The spontaneous light force exerted on atoms by scattering photons from resonant laser light is widely used in cooling and trapping experiments [1]. Its magnitude cannot exceed the fundamental limit $\hbar k \gamma$, where $\hbar k$ is the photon momentum and $\gamma$ is the maximum scattering rate for a saturated optical transition with natural linewidth $2 \gamma$. This upper limit of the force sets a lower limit for the distance required for the deceleration of atomic beams $[2,3]$, which typically is on the order of $1 \mathrm{~m}$ for thermal initial velocities.

It is well known that, in intense light fields, a much stronger force can result from a directed photon transfer between counterpropagating laser beams; the force is induced by cycles of absorption from one wave and stimulated emission into the other one [4,5]. For an ideal, i.e., perfectly directed photon transfer, the corresponding transfer rate is given by the optical Rabi frequency. In practice, the magnitude of the force is limited by the efficiency of the photon transfer and the available laser intensity.

A single high-intensity standing wave is a very basic and well-studied situation with respect to stimulated light forces $[4,6,7]$. Transverse cooling of an atomic beam has been demonstrated in an intense blue-detuned standing wave by Aspect et al. [8], and a red-detuned standing wave was used by Prentiss and Cable to slow down an atomic beam [9]. In the latter experiment, the average calculated slowing force did not exceed $2 \hbar k \gamma$ despite the very high Rabi frequencies of up to $\sim 400 \gamma$ applied in tightly focused beams. As we will explain, a single monochromatic standing wave cannot provide an efficient photon transfer over a broad velocity range.

We use a bichromatic field of two collinearly superposed standing waves. They have equal intensities and their frequencies $\omega_{0} \pm \Delta$ are symmetrically detuned with respect to an optical transition [Fig. 1(a)]. This produces a stimulated force with nearly ideal properties for atomicbeam slowing: (a) it works for a two-level system, (b) its magnitude is close to the ideal force resulting from the perfectly directed transfer of one photon per Rabi cycle, and (c) its velocity dependence is of nearly rectangular shape, combining a large width with abrupt flanks that well define the final slowing velocity. In this Letter, we present a continuous short-distance deceleration of a cesium atomic beam achieved with this "stimulated bichromatic force." The slowing force exceeds the maximum spontaneous force by a factor of $\sim 10$ and acts within a large velocity capture range of $\sim 50 \gamma / k$.

A physical interpretation of the force, as first pointed out by Voitsekhovich et al. [10], can be given using the old idea of photon transfer induced by resonant optical $\pi$ pulses $[4,11,12]$ : The light field can be viewed as two counterpropagating trains of resonant beat pulses, $A$ and $B$ [Fig. 1(a)]. When the $\pi$-pulse condition is fulfilled, i.e., when the Rabi frequency $\Omega_{R}$ associated with a single monochromatic traveling wave fulfills $\Omega_{R}=(\pi / 4) \Delta$, the effect of each beat pulse is to invert the atomic population. The pulse trains can thus force an atom to cycle between its lower and upper state in such a way that it repeatedly absorbs photons from one wave and emits them into the other one; the corresponding transfer rate is given by the beat frequency $1 / T=\Delta / \pi$ and the force is ideally $2 \hbar k / T[13]$.

(a)

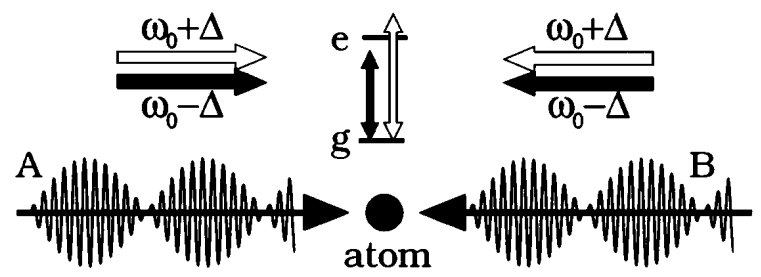

(b)

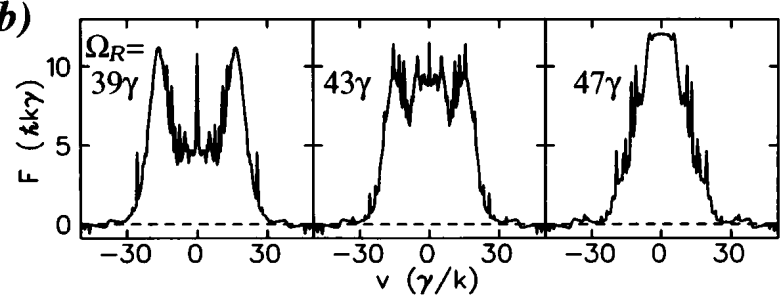

FIG. 1. (a) Light-field configuration for the stimulated bichromatic force. (b) Theoretical force as a function of the atomic velocity for $\Delta=39 \gamma, \phi=90^{\circ}$, and three different Rabi frequencies. The narrow spikes are due to velocity-tuned "Doppleron" resonances. 
Because of spontaneous emission, the relative beat phase $\phi=2 \pi \tau / T$ determines the favored direction of photon transfer; here $\tau$ is the time between a pulse from $A$ and a subsequent pulse from $B$. Spontaneous emission interrupts the repeated cycling in each transfer direction and inverts the direction of the momentum transfer. This occurs at an average rate $2 \gamma p_{e}$ where $p_{e}$ is the timeaveraged population probability of the excited state. For an asymmetric phase choice $(\phi \neq 0, \pi), p_{e}$ differs for both transfer directions. In an average over a time greater than $1 / \gamma$, repeated photon transfer in one direction is then favored. The result is an average directed force. An increase of the $\pi$-pulse repetition rate $1 / T \propto \Omega_{R}$ leads to more transfer cycles between two spontaneous emissions and thus to a higher, nonsaturating average force. For sinusoidal beat pulses, the optimum force with a magnitude of about $\hbar k / T$ is found for $|\phi| \approx \pi / 2$. This case represents the best compromise between the required asymmetry and the unwanted mutual pulse overlap.

The stimulated bichromatic force can alternatively be interpreted in terms of dipole force rectification $[14,15]$. Forces of this class were studied in several experiments for a variety of excitation schemes [16-20], mostly by atomic-beam deflection.

We have calculated the velocity-dependent force (averaged over one wavelength) by numerically solving the optical Bloch equations for an atom moving in the light field. Typical force profiles are shown in Fig. 1(b). An optimum combination of large force and broad velocity range is found for $1.0 \Delta \lesssim \Omega_{R} \lesssim 1.2 \Delta$, which is close to the $\pi$ pulse condition. The essential features of these profiles can be illustratively explained in a bichromatic dressedatom approach $[13,15,21]$. In particular, the large width (on the order of $2 \Delta / k$ ) of the velocity interval where a strong force occurs is interpreted in terms of an exceptional mechanism of energy transfer between atom and light field: The kinetic energy of a decelerated atom is transferred to the light field by a stimulated conversion of photons from one frequency component into the other, which occurs with a rate of up to $\sim \Omega_{R / 2 \pi}$. The light field can thus provide a power for acceleration $d E_{\mathrm{kin}} / d t=F v$ on the order of $\hbar \Delta \Omega_{R / \pi}$. In a monochromatic standing wave field as well as in all other considered bichromatic schemes $[8,9,16-20]$ the atomic energy is carried away by transforming laser photons into frequency-shifted photons [7] emitted spontaneously with a rate of only $\sim \gamma$. Since the average frequency shift of the emitted photons is limited by the spatial modulation of the light shifts, the power for acceleration $F v$ is limited to $\hbar \Omega_{R} \gamma$. As a consequence, in a single standing wave a strong stimulated force $\sim \hbar k \Omega_{R}$ is restricted to velocities $|v| \lesssim \gamma / k$.

In our experiments (Fig. 2), we use this stimulated bichromatic force to continuously decelerate a thermal atomic beam. We therefore apply the force in a reference frame moving with a velocity $v_{c}$ in the atomicbeam direction so that it covers a substantial part of the Maxwellian distribution. This is accomplished by shifting the two frequencies in the laser beam counterpropagating with the atomic beam down by $k v_{c}$ and those in the copropagating laser beam up by $k v_{c}$. A two-level system is realized with the Cs transition $6^{2} S_{1 / 2}\left(F=4, m_{F}=4\right) \rightarrow$ $6^{2} P_{3 / 2}\left(F^{\prime}=5, m_{F^{\prime}}=5\right)$ at $852 \mathrm{~nm}$ and $\sigma^{+}$-polarized light; the natural linewidth is $2 \gamma=2 \pi \times 5.3 \mathrm{MHz}$.

The four laser frequencies $\omega_{0} \pm \Delta \pm k v_{c}$ required for the stimulated slowing force are generated from the 600 $\mathrm{mW}$ output of a single-mode Ti:sapphire laser stabilized to $\omega_{0}+\Delta+k v_{c}$. The main beam is split into two parts, one of which is shifted by $-2 \Delta$ in a $200-\mathrm{MHz}$ acousto-optic modulator (AOM). The two beams are then recombined in a polarizing beam splitter and coupled into a polarization-maintaining monomode optical fiber for perfect mode matching. After the fiber the polarizations of the two superposed beams are rotated to $\pm 45^{\circ}$ with respect to the vertical axis and split by a polarizing beam splitter. The two outgoing beams are linearly polarized and contain equal parts of both frequency components $\omega_{0} \pm \Delta+k v_{c}$.

The first of these two bichromatic beams passes through a second $200-\mathrm{MHz}$ AOM, which shifts its two frequency components by $-2 k v_{c}$ to $\omega_{0} \pm \Delta-k v_{c}$. The beam is then superposed antiparallel with the atomic beam. The second bichromatic beam $\left(\omega_{0} \pm \Delta+k v_{c}\right)$ passes through an optical delay line for varying the relative beat phase $\phi$. It is then sent through the rear window of the Cs oven and superposed parallel to the atomic beam. Both beams have a diameter $\left(1 / e^{2}\right.$ intensity drop) of $2.5 \mathrm{~mm}( \pm 5 \%)$. The power in each of the four frequency components can be as high as $50 \mathrm{~mW}$.

The atomic beam is formed by a 2-mm oven aperture and another 2-mm aperture $11 \mathrm{~cm}$ apart. The oven is operated at a temperature of $80{ }^{\circ} \mathrm{C}$ and its rear window is heated to $120{ }^{\circ} \mathrm{C}$ to avoid Cs deposition. The absorption in the oven is smaller than $5 \%$ because the optical path inside the oven is short $(5 \mathrm{~mm})$ and an axially magnetized

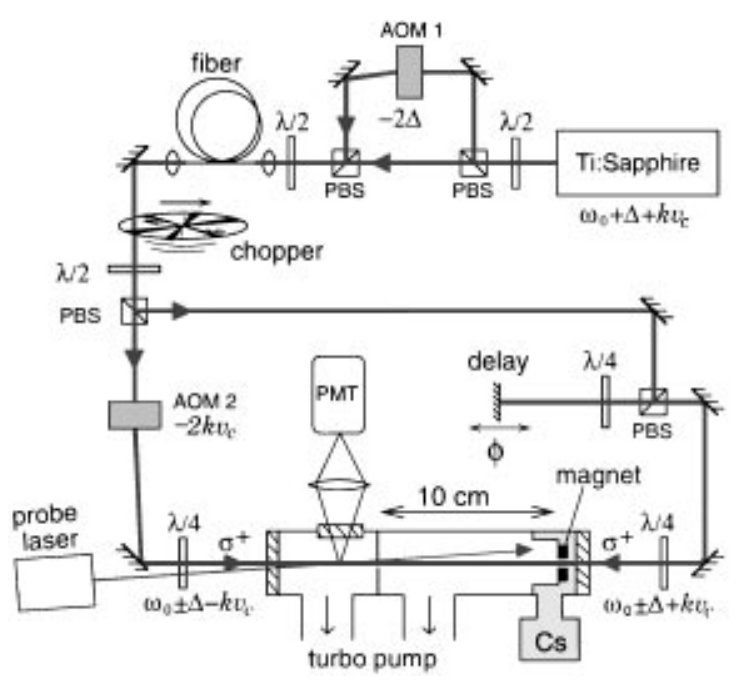

FIG. 2. Experimental setup; for details, see text. 
rare-earth ring magnet is used to locally tune the atoms out of resonance.

We measure the longitudinal atomic velocity distribution with a $\sigma^{+}$-polarized $10-\mu \mathrm{W}$ probe laser beam from a narrow-linewidth distributed-Bragg-reflector diode laser (SDL-5712). The beam is overlapped with the atomic beam under a small angle of $50 \mathrm{mrad}$ and its frequency is slowly tuned over the velocity profile. The fluorescence intensity, which is proportional to the density of atoms at the Doppler-selected speed, is detected $1.5 \mathrm{~cm}$ behind the second aperture with a photomultiplier tube. Because of the large probe beam diameter $(3.5 \mathrm{~mm})$, the atomic beam is probed over its entire cross section. Every $500 \mu \mathrm{s}$ a chopper wheel blocks the strong slowing beams for short $100-\mu$ s intervals, during which we detect and average the probe-induced fluorescence. The duty cycle for deceleration of 0.8 multiplied by the distance $12.5 \mathrm{~cm}$ between the oven and the detection region gives an effective slowing distance of $\sim 10 \mathrm{~cm}$.

A 50-mW $\sigma^{+}$-polarized repumping laser beam issued from a second diode laser (not shown in Fig. 3) is overlapped with the counterpropagating atomic beam under a small angle of $\sim 5 \mathrm{mrad}$. Its frequency is tuned to the $F=3 \rightarrow F^{\prime}=3,4$ transitions and broadened over the Doppler width of the atomic beam $(\sim 500 \mathrm{MHz})$ by modulating the diode current at $2 \mathrm{MHz}$.

To first demonstrate the efficiency of the bichromatic force, we choose a center velocity $v_{c}$ of $175 \mathrm{~m} / \mathrm{s}$, i.e., in the vicinity of the maximum of the Maxwell-Boltzmann

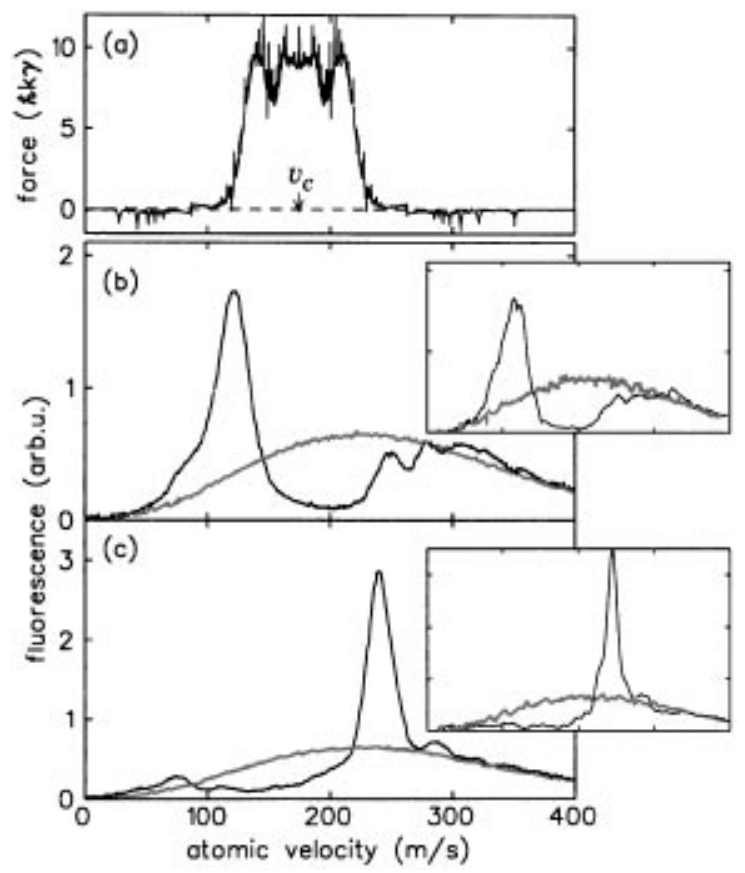

FIG. 3. (a) Representative force profile for $v_{c}=175 \mathrm{~m} / \mathrm{s}$, $\Delta=39 \gamma, \Omega_{R}=43 \gamma$. (b) Measured atomic-beam velocity distribution with (black) and without (grey) stimulated force for $\phi=90^{\circ}, v_{c}=175 \mathrm{~m} / \mathrm{s}, \Delta=39 \gamma$, and $40 \mathrm{~mW}$ per singlefrequency traveling wave. (c) Same as before, but for $\phi=$ $-90^{\circ}$. Insets: Monte Carlo simulations of the experiment. distribution $(220 \mathrm{~m} / \mathrm{s})$. With a power of $40 \mathrm{~mW}$ per single frequency component ( $160 \mathrm{~mW}$ in total) and a beat frequency $2 \Delta=2 \times 39 \gamma=2 \pi \times 205 \mathrm{MHz}$, the velocity capture range is $120 \mathrm{~m} / \mathrm{s}$. Continuous deceleration is demonstrated in Fig. 3(b): Atoms with a velocity below $240 \mathrm{~m} / \mathrm{s}$ are slowed to $120 \mathrm{~m} / \mathrm{s}$ within only $10 \mathrm{~cm}$. The emptied velocity region coincides with the region where the stimulated force is large, as can be seen by comparison with Fig. 3(a). By displacing the delay mirror by $\pi c / 4 \Delta=36.5 \mathrm{~cm}$, we change the relative beat phase $\phi$ from $90^{\circ}$ to $-90^{\circ}$. This inverts the direction of the stimulated force and produces a clear acceleration of the atoms [Fig. 3(c)].

We have performed simple Monte Carlo simulations to check that these measurements can be completely understood on the basis of calculated force profiles. We have taken into account the variation of the Rabi frequency across the Gaussian laser beam profile $\left(\Omega_{R}=57 \gamma\right.$ in the beam center and $35 \gamma$ at the edge of the apertures) and the variation of the beat phase $\phi$ between $\sim 60^{\circ}$ and $\sim 120^{\circ}$ along the atomic beam [21]. As shown in Fig. 1(b), the shape of the force profile changes significantly with $\Omega_{R}$ around its optimum value. This somewhat affects the magnitude of the applied force and also the final slowing velocity. Longitudinal momentum diffusion is modeled as a velocity-independent process with a corresponding diffusion coefficient of $0.5 \hbar^{2} k^{2} \Omega_{R}^{2} / \gamma$. Transverse diffusion due to stimulated and spontaneous processes is negligible because of the large laser beam diameter and the short deceleration length.

The results of these simulations performed with 10000 atoms per trace are shown in the insets of Fig. 3. They agree very well with the corresponding measurements: the position, height, and width of the cooled peak and even details of the velocity distribution are well reproduced. The experimental signals therefore contain no significant contributions of spurious effects, such as a residual spontaneous force or optical pumping. The simulations further indicate that the few atoms left inside the resonance region of the force result from the deviation from the optimum matching of $\Omega_{R}$ to $\Delta$ in some parts of the Gaussian laser beam cross section. The spatial variation of $\Omega_{R}$ also gives the dominant contribution to the observed widths of the cooled velocity distributions.

In systematic measurements we have studied the dependence of the slowed atomic distribution on the beat phase, the detuning of the mean frequency from $\omega_{0}$, the total laser power, and the power imbalance between the beams [21]. The force is insensitive against deviations from the ideal parameters by $\pm 10^{\circ}$ in the beat phase, $\pm 25 \mathrm{MHz}$ in the mean frequency, and $\pm 20 \%$ in the total power and power balance. These experimental results agree well with corresponding calculated force profiles.

By reducing the center velocity $v_{c}$ down to $\sim 100 \mathrm{~m} / \mathrm{s}$ (Fig. 4), we have produced a continuous beam of slow atoms with velocities in the $10-\mathrm{m} / \mathrm{s}$ range, well within the capture velocity of a standard magneto-optical trap. 
The measurements in Fig. 4, taken with $2 \Delta=2 \pi \times$ $225 \mathrm{MHz}$, demonstrate the slowing from $\sim 180 \mathrm{~m} / \mathrm{s}$ down to $27 \mathrm{~m} / \mathrm{s}$ [Fig. 4(a)] and from $\sim 160 \mathrm{~m} / \mathrm{s}$ down to $8 \mathrm{~m} / \mathrm{s}$ [Fig. 4(b)]. To optimize the flux of slow atoms we even had to reduce the slowing force by using a power of only $25 \mathrm{~mW}$ per single-frequency component. This is because, for a full force of $10 \hbar k \gamma$, the stopping distance for atoms initially at $160 \mathrm{~m} / \mathrm{s}$ is less than $3 \mathrm{~cm}$, much shorter than the distance between the oven and the observation region in our present apparatus.

We now estimate the flux of slowed atoms produced by our method. The total atomic flux of the uncooled Maxwell-Boltzmann distribution through the second 2-mm diaphragm is $5 \times 10^{10}$ atoms/s for an oven temperature of $80^{\circ} \mathrm{C}\left( \pm 10^{\circ} \mathrm{C}\right)$. The flux of slowed atoms with $v \leq$ $35 \mathrm{~m} / \mathrm{s}$ is $5 \times 10^{8}$ atoms $/ \mathrm{s}$ [Fig. 4(a)]. According to our Monte Carlo simulations, this flux can be substantially increased by using a stopping distance of only $3-5 \mathrm{~cm}$ and by raising the Cs pressure in the oven by a factor of 10 $\left(110{ }^{\circ} \mathrm{C}\right)$. A loading rate for a magneto-optical trap of a few $10^{10}$ atoms/s, similar to the highest loading rates obtained with the Zeeman slowing method [1], is realistic under the excellent vacuum conditions required for evaporative cooling and Bose-Einstein condensation [22-24].

We have demonstrated a very efficient method to produce continuous, intense, and slow atomic beams. The rapid progress in high-power solid-state laser devices will allow one to produce even larger velocity capture ranges and larger forces, since they both scale approximately as the optical Rabi frequency. The unique properties of the stimulated bichromatic force make it interesting for many other applications in atom manipulation, atom lithography [25], and atom optics [26], such as slowing of rare gases or other, exotic elements with weak or not completely closed transitions [27], the transverse collimation of dense, strongly divergent atomic beams, the

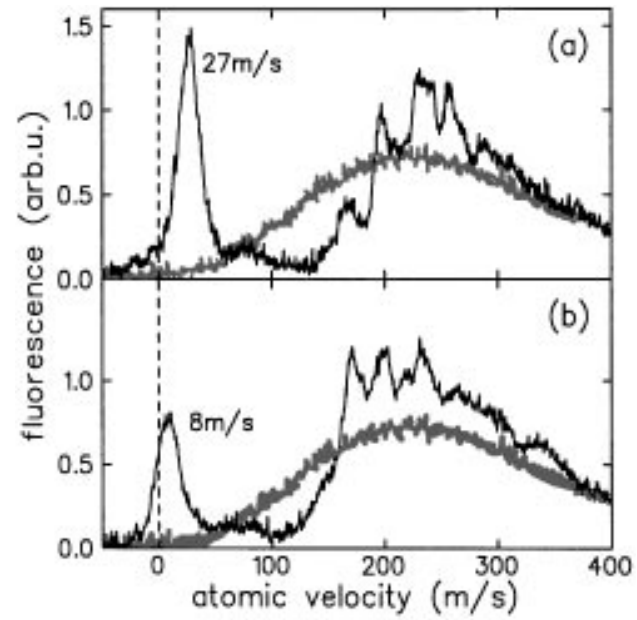

FIG. 4. Measured atomic velocity distributions with and without stimulated force. (a) $v_{c}=110 \mathrm{~m} / \mathrm{s}$ and (b) $v_{c}=$ $91 \mathrm{~m} / \mathrm{s}$. large-angle atomic-beam deflection, or the laser cooling of fast ion beams in storage rings [28].

We thank A. Fioretti for his help in the early stages of the experiment, G. Wassik and M. Zielonkowski for assistance, and D. Schwalm and D. Habs for encouragement and support. Yu. B. O. is indebted to the Alexander von Humboldt-Stiftung for support. This work was partly supported by the Deutsche Forschungsgemeinschaft. Laboratoire Kastler Brossel is an unité associée au CNRS et à l'Université Pierre et Marie Curie.

*Present address: Institute d'Optique Théorique et Appliquée, Orsay, France.

[1] H. Metcalf and P. van der Straten, Phys. Rep. 244, 203 (1994).

[2] J. Prodan et al., Phys. Rev. Lett. 54, 992 (1985).

[3] W. Ertmer et al., Phys. Rev. Lett. 54, 996 (1985).

[4] A. P. Kazantsev, Sov. Phys. JETP 39, 784 (1974).

[5] I. Nebenzahl and A. Szöke, Appl. Phys. Lett. 25, 327 (1974).

[6] J. P. Gordon and A. Ashkin, Phys. Rev. A 21, 1606 (1980).

[7] J. Dalibard and C. Cohen-Tannoudji, J. Opt. Soc. Am. B 2, 1707 (1985).

[8] A. Aspect et al., Phys. Rev. Lett. 61, 826 (1988).

[9] M. Prentiss and A. Cable, Phys. Rev. Lett. 62, 1354 (1989).

[10] V.S. Voitsekhovich et al., Sov. Phys. Tech. Phys. 33, 690 (1988); JETP Lett. 49, 161 (1989); Sov. Phys. JETP 72, 219 (1991). Because of a marked mismatch between $\Omega_{R}$ and $\Delta$ the observed force was $\lesssim 1 \hbar k \gamma$.

[11] H. Friedman and A. D. Wilson, Appl. Phys. Lett. 28, 270 (1976).

[12] B. Nölle et al., Europhys. Lett. 3, 261 (1996).

[13] R. Grimm, J. Söding, and Yu. B. Ovchinnikov, Opt. Lett. 19, 658 (1994).

[14] A.P. Kazantsev and I. V. Krasnov, JETP Lett. 46, 332 (1987).

[15] R. Grimm et al., in Proceedings of the International School of Physics "Enrico Fermi" Course CXXXI, Varenna, 1995 (IOS, Amsterdam, 1996).

[16] R. Grimm et al., Phys. Rev. Lett. 65, 1415 (1990); Yu. B. Ovchinnikov et al., Opt. Commun. 102, 155 (1993). In this work the force was restricted to velocities $|v| \lesssim \gamma / k$.

[17] P. R. Hemmer et al., Phys. Rev. Lett. 68, 3148 (1992).

[18] J. Söding et al., Europhys. Lett. 20, 101 (1992).

[19] R. Gupta et al., Phys. Rev. Lett. 71, 3087 (1993).

[20] T. T. Grove et al., Phys. Rev. A 51, R4325 (1995).

[21] J. Söding, MPI für Kernphysik Report No. MPI H - V22 1996, Heidelberg, 1996.

[22] M. H. Anderson et al., Science 269, 198 (1995).

[23] C. C. Bradley et al., Phys. Rev. Lett. 75, 1687 (1995).

[24] K. B. Davis et al., Phys. Rev. Lett. 75, 3969 (1995).

[25] J. J. McClelland et al., Science 262, 877 (1993).

[26] Special issue on Optics and Interferometry with Atoms [Appl. Phys. B 54, 319-485 (1992)].

[27] G. M. Tino, Comments At. Mol. Phys. 29, 5 (1993).

[28] H.-J. Miesner et al., Phys. Rev. Lett. 77, 623 (1996). 